\title{
Geodinâmica do Manto em Bordas de Margens Divergentes
}

\author{
Edgar Bueno dos Santos (IAG/USP), Victor Sacek (IAG/USP)
}

Copyright 2016, SBGf - Sociedade Brasileira de Geofísica

Este texto foi preparado para a apresentação no VII Simpósio Brasileiro de Geofísica. Ouro Preto, 25 a 27 de outubro de 2016. Seu conteúdo foi revisado pelo Comitê Técnico do VII SimBGt, mas não necessariamente representa a opinião da SBGf ou de seus associados. É proibida a reprodução total ou parcial deste material para propósitos comerciais sem prévia autorização da SBGf.

\begin{abstract}
In this work we study the mantle dynamics in continental margins by using numerical models capable of simulating the conductive and convective processes present in the mantle. Initially, we performed simulations in order to obtain an initial thermal structure where the thickness of the continental lithosphere is stabilized. In these simulations, we evaluate the influence of some parameters such as the initial viscosity, the activation energy and the activation volume, choosing the best scenarios to represent the real continental lithosphere. Using the obtained thermal structure we performed new simulations to study the thermal subsidence of divergent margins taking into account the convective processes in the asthenosphere. Finally, we discuss about the convective effects present in the asthenospheric mantle comparing them with purely conductive models.
\end{abstract}

\section{Introdução}

Pode-se diferenciar a subsidência de bacias sedimentares ao longo de margens divergentes, como as presentes ao longo da costa brasileira entre subsidência tectônica e a subsidência total. A subsidência total é a soma da subsidência tectônica mais a subsidência devida à carga do pacote sedimentar derivado da erosão dos continentes e sua deposição ao longo da costa. Já a subsidência tectônica é a subsidência originada por mecanismos tectônicos e independe de carga sedimentar sobre a crosta terrestre (Stüwe, 2002), como processos térmicos, topografia dinâmica e variações na espessura crustal.

A primeira explicação satisfatória para a existência da subsidência tectônica foi dada por McKenzie (1978). Em seu modelo ele propôs que a subsidência tectônica ocorre pelo resfriamento da litosfera devido à perda de calor para a superfície após o seu afinamento devido ao estiramento litosférico uniforme. Com o esfriamento térmico, o manto astenosférico sob a litosfera, que tem um comportamento fluido no tempo geológico, resfria-se em contato com a base da litosfera, podendo ser agregado a sua base, tornando-se mais rígida e resultando no espessamento da litosfera. Através deste modelo foi possível prever também o fluxo térmico do embasamento para a bacia sedimentar ao longo do tempo, informação fundamental para a avaliação do potencial petrolífero de uma bacia, o que consagrou o modelo na indústria de petróleo.
Entretanto, o modelo de McKenzie assume que a condução de calor ocorre exclusivamente na direção vertical, desprezando-se efeitos laterais de condução térmica. Steckler \& Watts (1980) mostraram que a variação lateral da espessura da litosfera pode induzir mudanças expressivas na evolução térmica da litosfera e consequentemente na bacia sedimentar.

Outro processo que controla a subsidência de margens divergentes é a convecção do manto astenosférico abaixo da litosfera, cujo vigor é dependente da viscosidade. Variações laterais na espessura da litosfera ao longo das margens continentais podem induzir células de convecção de pequena escala na astenosfera (King \& Anderson, 1998), resultando no aumento do fluxo térmico para a base da litosfera afinada (Lucazeau, 2008).

Recentemente alguns trabalhos mostraram como a geometria 3D da margem pode influenciar a história de subsidência das bacias marginais (Farrington et al., 2010; Braun et al., 2010; Sacek \& Ussami, 2013). Sacek \& Ussami (2013) mostraram como a curvatura da margem, como observado no sudeste do Brasil, poderia induzir subsidência diferencial da margem devido ao efeito combinado da condução térmica em 3D e processos convectivos no manto. Sacek \& Ussami (2013) mostraram que parte da subsidência diferencial da margem sudeste do Brasil pode ter ocorrido devido ao resultado da curvatura da margem e a dinâmica interna do manto.

O presente trabalho tem como objetivo avaliar diferentes configurações físicas e geométricas de margem continentais para avaliar a influência desses parâmetros na história de subsidência e de fluxo térmico basal para as bacias sedimentares ao longo da margem.

\section{Estabilidade da Litosfera Continental}

Doin et al. (1997) mostraram que a espessura em que a litosfera, tanto oceânica quanto continental, se estabiliza está intimamente relacionada com o número de Rayleigh e a reologia utilizada. Eles também observaram que convecções desencadeadas por variações laterais na espessura da litosfera, aos poucos desestabilizavam a litosfera espessa.

Desse modo, é importante a obtenção de um modelo onde se tenha a garantia de que a configuração térmica inicial para litosfera não estirada esteja em equilíbrio, não sofrendo variações significativas na espessura para intervalos de tempo inferiores à idade máxima de margens divergentes (<200 Ma).

Foram realizados diversos modelos numéricos utilizandose o software CitcomCU (Moresi \& Gurnis, 1996; Zhong, 2006), um código computacional construído para simular processos convectivos no manto terrestre em 3D, a fim de se avaliar a influência dos parâmetros que governam 
as equações da dinâmica do manto, como a viscosidade da base $\eta_{0}$, a energia de ativação $E$ e o volume de ativação $V$.

Em todos os testes apresentados aqui se considerou um modelo de caixa simples, com a base do modelo a uma temperatura de $1800^{\circ} \mathrm{C}$ e o topo a $0^{\circ} \mathrm{C}$. Foi adotada uma profundidade de $660 \mathrm{~km}$, e para o comprimento utilizouse o dobro da profundidade. Os modelos foram feitos com um gride de $33 \times 3 \times 33(x, y, z)$ num total de 3267 nós. A escolha de apenas três nós na direção y foi feita a fim de que se observasse o comportamento do modelo apenas em duas dimensões.

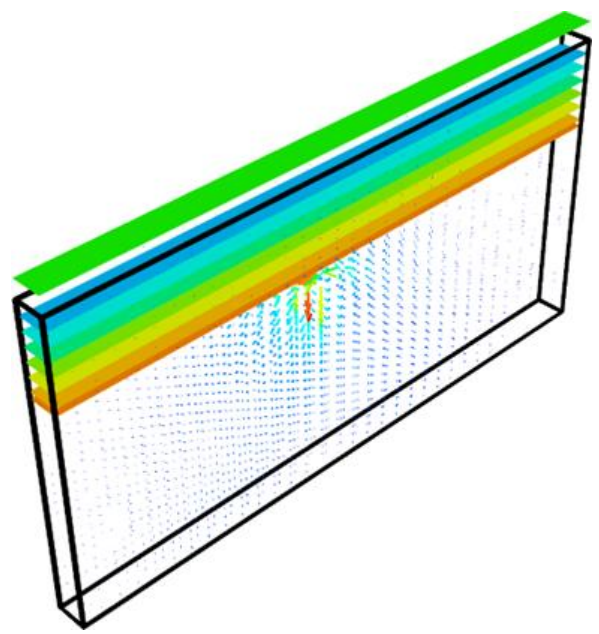

Figura 1 - Configuração do perfil de temperatura inicial utilizado para os modelos. A base se encontra a uma temperatura de $1800^{\circ} \mathrm{C}$. Foi utilizado um grid de 3267 nós.

A Figura 1 apresenta a configuração de temperatura inicial utilizada em nossos modelos. Foi adotada uma variação linear da temperatura na parte superior do modelo (aproximadamente $80 \mathrm{~km}$ de profundidade) e uma temperatura constante até a base do modelo. A porção superior representa a espessura inicial da litosfera. Foi introduzida uma pequena perturbação no centro do modelo, esta perturbação é necessária para garantir que o modelo convirja, visto que o perfil de temperatura adotado é puramente horizontal sem a perturbação não seria possível dar início aos processos convectivos no modelo.

Foram realizados testes para diversos valores de viscosidade, energia de ativação e volume de ativação. No total foram simulados 60 modelos diferentes utilizando-se todas as combinações possíveis entre os valores para os três parâmetros apresentados na Tabela 1.

Para determinar a espessura em que a litosfera se estabiliza é necessário encontrar o limite térmico entre a litosfera e a astenosfera. Entretanto, este limite não é bem definido na literatura, e usualmente é assumido como a isoterma de $1400 \mathrm{~K}$. Neste trabalho este limite será assumido como sendo a isoterma de $1300^{\circ} \mathrm{C}$.
Tabela 1 - Valores utilizados para os modelos numéricos. Foram realizados modelos com todas as combinações possíveis entre esses três parâmetros. Os valores de $E$ e $V$ apresentados são adimensionais.

\begin{tabular}{ccc}
\hline $\mathbf{E}$ & $\mathbf{V}$ & $\boldsymbol{\eta}_{\mathbf{0}}$ (Pa.s) \\
\hline 30 & 0 & $5 \times 10^{20}$ \\
20 & 5 & $4 \times 10^{20}$ \\
- & 7.5 & $3 \times 10^{20}$ \\
- & 10 & $1 \times 10^{20}$ \\
- & 12.5 & $5 \times 10^{19}$ \\
- & 15 & -
\end{tabular}

De modo que fosse possível realizar uma comparação entre todos os modelos estimou-se a profundidade da isoterma de $1300^{\circ} \mathrm{C}$ para todos os modelos em $500 \mathrm{Myr}$ após o início da simulação numérica. A Figura 2 abaixo apresenta esta comparação.

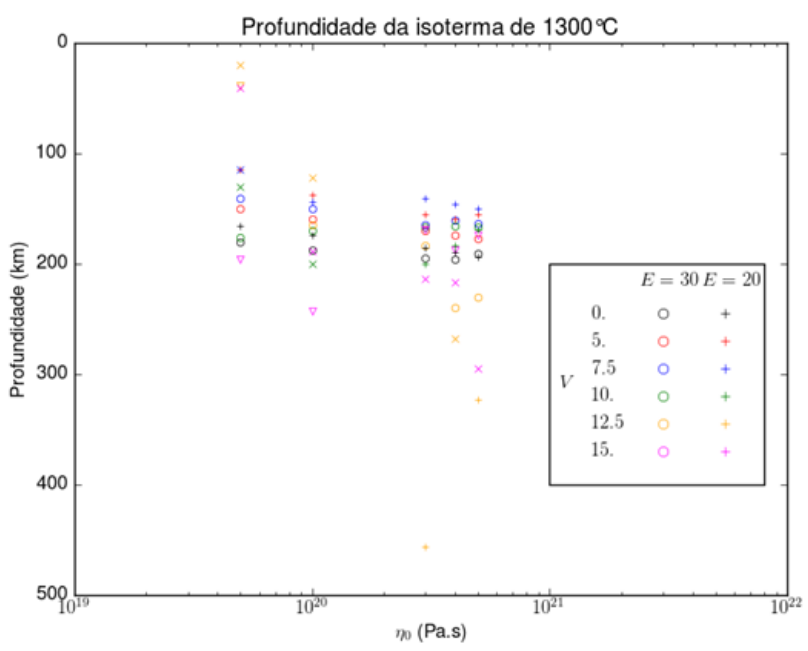

Figura 2 - Profundidade da isoterma de $1300^{\circ} \mathrm{C}$ para todos os modelos. Os pontos marcados por um "x" e com triângulo são pontos com $E=20$ e $E=30$ respectivamente, onde o modelo não chegou a 500 Myr.

Pode-se verificar através da Figura 2 que valores menores para a viscosidade da base implicam em uma menor profundidade para a isoterma de $1300^{\circ} \mathrm{C}$. É possível também notar que valores menores para a energia de ativação resultam em um afinamento da litosfera, isto ocorre, pois esta energia atua como uma barreira potencial, dificultando a existência de processos convectivos no manto. Por fim, analisando o volume de ativação, observa-se dois comportamentos distintos. Para valores muito baixos ocorre um espessamento da litosfera, pois a viscosidade no interior do modelo se torna muito alta dificultando a ocorrência de processos convectivos. Por outro lado, quando utilizamos valores 
muito altos para o volume de ativação a isoterma de $1300^{\circ} \mathrm{C}$ também será mais profunda. Entretanto, neste caso a viscosidade do modelo é tão baixa que a base da camada térmica limite ocorre a uma temperatura muito mais baixa do que a encontrada entre 0 limite litosfera/astenosfera, o que contradiz as estimativas encontradas na literatura.

\section{Estiramento da Litosfera}

Para simularmos cenários no contexto de margens divergentes, é necessário que em um dado instante de tempo a litosfera sofra um estiramento.

Tal estiramento deve ser feito de forma que a base da porção estirada da litosfera mantenha a mesma temperatura da base da litosfera não estirada. Desse modo, podemos realizar o estiramento da litosfera através de uma transformação linear da seguinte forma

$$
\begin{aligned}
& T(h)=T_{0}(h \beta), \quad 0 \leq h<\frac{L}{\beta} \\
& T(h)=T_{0}\left(h^{\prime}\right), \quad \frac{L}{\beta} \leq h<H
\end{aligned}
$$

onde $T(h)$ é a temperatura após aplicada a transformação, $T_{0}(h)$ é a temperatura da estrutura térmica não estirada, $h$ é a profundidade, $H$ é a profundidade total do modelo, $\beta$ é o fator de estiramento, $L$ é a espessura da litosfera não estirada e $h^{\prime}$ é dado por

$$
h^{\prime}=\frac{H-L}{H-\frac{L}{\beta}}\left(h-\frac{L}{\beta}\right)+L
$$

A Figura 3 é uma representação esquemática de como essa transformação modifica a estrutura térmica inicial. $O$ ponto em vermelho representa um mesmo ponto da isoterma, antes e após o estiramento.

Para podermos avaliar os efeitos da condução térmica e da convecção mantélica, foram simulados diversos cenários numéricos em uma representação bidimensional no contexto de margens divergentes. Para que a comparação entre os modelos apresentados neste trabalho e os modelos apresentados por McKenzie (1978) e Steckler \& Watts (1980) seja possível, é necessário utilizar o mesmo perfil do fator de estiramento $\beta$ utilizado por Steckler \& Watts (1980), e a mesma espessura de litosfera utilizada por McKenzie (1978) e Steckler \& Watts (1980). A Figura 4 apresenta o perfil de estiramento utilizado por Steckler \& Watts (1980). Diferentemente do perfil utilizado por McKenzie (1978), este modelo não apresenta um perfil de estiramento uniforme, assumindo valores diferentes de $\beta$ para cada um dos nós. O espaçamento entre os nós é de aproximadamente $10 \mathrm{~km}$.

Através da Figura 2 é possível identificar os modelos que obtiveram espessura da litosfera da ordem de $125 \mathrm{~km}$ conforme foi utilizado por McKenzie (1978) e Steckler \& Watts (1980). Neste trabalho optou-se por utilizar todos os modelos que apresentaram espessura da litosfera entre $110 \mathrm{~km}$ e $165 \mathrm{~km}$.


Figura 3 - Representação esquemática da isoterma da litosfera não estirada (acima) e a litosfera estirada (embaixo) através da equação 1 .

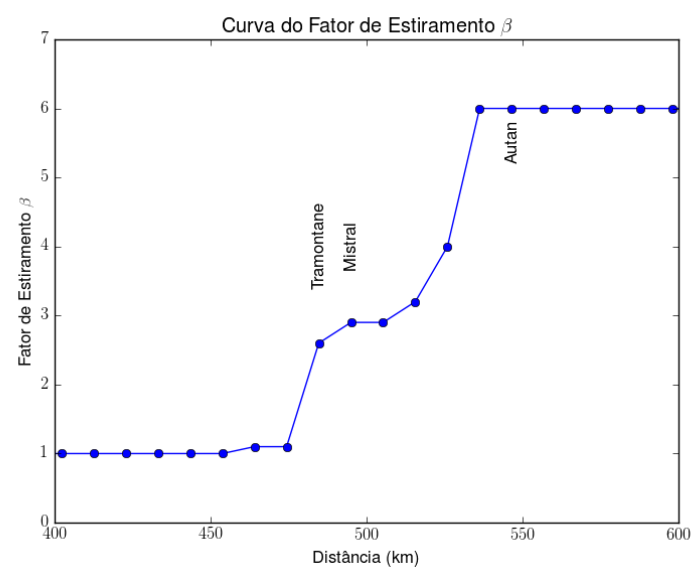

Figura 4 - Perfil do fator de estiramento $\beta$. Estão representadas as posições das três estações utilizadas por Steckler e Watts (1980). 
Foi necessário utilizar um gride mais refinado para os modelos para que fosse possível aplicar o estiramento litosférico conforme descrito pela equação 1. Assim, repetiu-se as simulações que apresentaram valores para a espessura da litosfera compatíveis com o utilizado por McKenzie (1978) e Steckler \& Watts (1980), para um gride de $65 \times 9 \times 65(x, y, z)$ num total de 38025 nós. A profundidade do modelo foi mantida em $660 \mathrm{~km}$ e a diferença de temperatura entre 0 topo e a base de $1800^{\circ} \mathrm{C}$. Para refinar ainda mais o modelo na direção $\mathrm{x}$, adotou-se o comprimento do modelo como sendo igual ao da profundidade.

\section{Taxa de Subsidência}

Para realizarmos a comparação entre os modelos obtidos neste trabalho, e os modelos de McKenzie (1978) e Steckler \& Watts (1980) é necessário calcularmos a taxa de subsidência obtida através das simulações.

O software CitcomCU calcula internamente um parâmetro conhecido como tração de superfície, e através deste parâmetro podemos calcular a taxa de subsidência para cada instante da simulação. Tal cálculo pode ser realizado através da seguinte equação

$$
w=\frac{\left(\eta_{\text {ref }} \kappa S \prime\right)}{H^{2}\left(\rho_{m}-\rho_{\text {sed }}\right)}
$$

onde $\eta_{0}$ é a viscosidade de referência, $\kappa$ é a difusividade térmica, $\rho_{m}$ e $\rho_{\text {sed }}$ são respectivamente a densidade do manto $\mathrm{e}$ a densidade dos sedimentos preenchendo a bacia, $g$ é a aceleração da gravidade, $H$ é a profundidade até a base do modelo e $S^{\prime}$ é a tração de superfície adimensional. Aqui consideraremos uma bacia preenchida com água, assim $\rho_{\text {sed }}=\rho_{w}$, onde $\rho_{w}$ é a densidade da água, e a equação 2 pode ser escrita como

$$
w=\frac{\left(\eta_{r e f} \kappa S^{\prime}\right)}{H^{2}\left(\rho_{m}-\rho_{w}\right)}
$$

Os valores das constantes utilizados nesses modelos estão apresentados na Tabela 2.

Tabela 2 - Valores dos parâmetros utilizados para o cálculo da subsidência.

\begin{tabular}{ll}
\hline$\rho_{m}$ & $3500 \mathrm{~kg} / \mathrm{m}^{3}$ \\
$\rho_{m}$ & $1000 \mathrm{~kg} / \mathrm{m}^{3}$ \\
$H$ & $660 \mathrm{~km}$ \\
$K$ & $10^{-6} \mathrm{~m}^{2} / \mathrm{s}$ \\
$\eta_{\text {ref }}$ & $5 \times 10^{20}$ Pa.s \\
\hline
\end{tabular}

A Figura 5 apresenta uma curva de subsidência ao longo do eixo $x$ para diversos valores de tempo. Podemos observar a presença de uma subsidência na região que sofreu estiramento litosférico bem como um soerguimento na porção offshore do rifte. A ocorrência deste soerguimento deve estar relacionada com convecções de pequena escala induzidas pelo gradiente térmico lateral como demonstrado por Buck (1986).

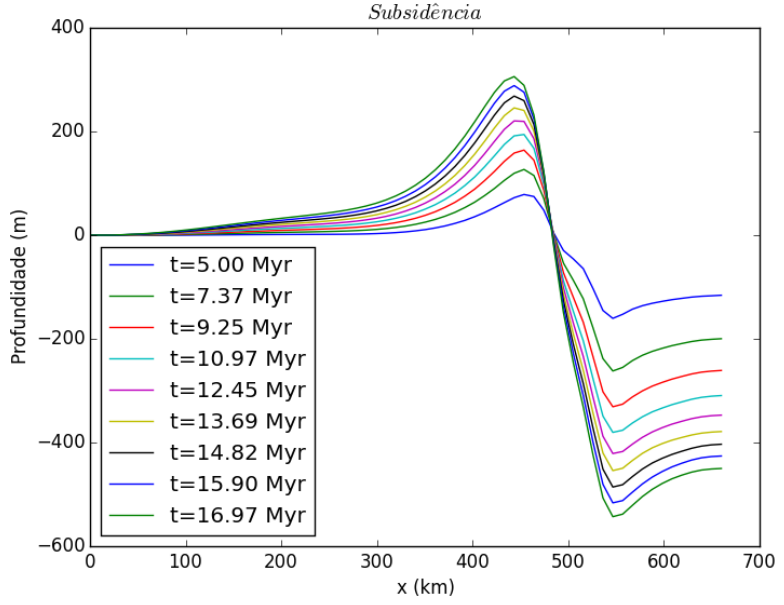

Figura 5 - Curva de subsidência em relação a posição em $x$ para um dado instante de tempo $t$.

$\mathrm{Na}$ figura 6 apresentamos uma comparação entre a subsidência obtido através dos nossos modelos com as obtidas por McKenzie (1978) e Steckler \& Watts (1980) para o poço de Autan localizado no Golfo do Leão. $E$ possível verificarmos a presença de oscilações com pequenos comprimentos de onda. Tais feições estão relacionadas com a topografia dinâmica no sentido de que a convecção sob a litosfera afinada induz tensões na base da mesma, deslocando-a para cima e para baixo ao longo do tempo. Quanto menor for a viscosidade da astenosfera maior será o vigor da convecção, induzindo maiores perturbações na litosfera. Essas perturbações podem gerar uma discordância erosiva modificando a evolução estratigráfica da bacia (Petersen et al, 2010).



Figura 6 - Comparação entre a subsidência para o poço de Autan, entre os modelos de Mckenzie (1978), Steckler e Watts (1980) e o modelo apresentado neste trabalho indicado com os quadrados vermelhos. 


\section{Discussão e Conclusões}

A incorporação de processos convectivos nas simulações da dinâmica do manto apresenta características que não são possíveis de se obter quando utilizamos modelos puramente condutivos. Vimos que o soerguimento da porção offshore da bacia apresentada na Figura 5 e as variações de pequeno comprimento de onda presentes na taxa de subsidência na Figura 6 são fenômenos que só podem ser obtidos quando levamos em consideração os processos de convecção mantélica.

Em todos os cenários observamos que a subsidência total ao longo do tempo geológico é inferior ao valor obtido em modelos puramente condutivos. Isto se deve ao fato de que a convecção astenosférica preserva por mais tempo a litosfera aquecida, atuando em oposição ao efeito de resfriamento por condução térmica (Sacek e Ussami, 2013). Assim, para que a taxa de subsidência seja compatível com a observada na margem divergente a espessura inicial da litosfera não estirada deve ser maior do que a prevista nos trabalhos anteriores.

Assim, através das simulações podemos verificar a importância da utilização de modelos mais complexos, que incorporem tanto a condução térmica em 3D como os processos convectivos presentes na astenosfera.

\section{Agradecimentos}

Edgar Bueno dos Santos agradece a ANP pela bolsa de mestrado concedida.

\section{Referências}

Buck W. R., Small-scale convection induced by passive rifting: the cause for uplift of rift shoulders, Earth and Planetary Science Letters, 1986, vol. 77, p. 362

Doin M.-P., Fleitout L., Christensen U., Mantle convection and stability of depleted and undepleted continental lithosphere, Journal of Geophysical Research, 1997, vol. 102, p.2771

McKenzie, D. (1978). Some remarks on the development of sedimentary basins. Earth and Planetary Science Letters, 40, 25-32.

Moresi, L. N. \& Gurnis, M. Constraints on lateral strength of slabs from 3-D dynamic flow models. Earth Planet. Sci. Lett. 138, 15-28 (1996)

Petersen, K. D., Nielsen, S. B., Clausen, O. R., Stephenson, R., Gerya, T. (2010). Small-Scale Mantle Convection Produces Stratigraphic Sequences in Sedimentary Basins. Science, 329, 827-830.

Sacek, V., \& Ussami, N. (2013). Upper mantle viscosity and dynamic subsidence of curved continental margins. Nature communications, 4.

Steckler, M. S., \& Watts, A. B. (1980). The Gulf of Lion: subsidence of a young continental margin. Nature, 287, 425-429.

Zhong, S. Constraints on thermochemical convection of the mantle from plume heat flux, plume excess temperature and upper mantle temperature. J. Geophys. Res. 111, B04409 (2006). 